\title{
Microstructural Evolution and Recrystallization Behavior Traced by Electron Channeling Contrast Imaging
}

\author{
Jin-Su Oh, Cheol-Woong Yang*
}

School of Advanced Materials Science \& Engineering, Sungkyunkwan University, Suwon 16419, Korea

*Correspondence to:

Yang CW

(iD) http://orcid.org/0000-0003-0475-8399

Tel: $+82-31-290-7362$

Fax: +82-31-290-7371

E-mail: cwyang@skku.edu

Received December 25, 2018

Revised December 27, 2018

Accepted December 27, 2018
Electron channeling contrast imaging (ECCI) is one of the imaging techniques in scanning electron microscopy based on a variation in electron backscattering yield depending on the direction of the primary electron beam with respect to the crystal lattice. The ECCI provides not only observation of the distribution of individual grains and grain boundaries but also identification of the defects such as dislocations, twins, and stacking faults. The ECCI at the interface between recrystallized and deformed region of shot peening treated nickel clearly demonstrates the microstructural evolution during the recrystallization including original grain boundaries, and thus can provide better insight into the recrystallization behavior.

Key Words: Electron channeling contrast imaging, Recrystallization, Shot peening, Original grain boundaries

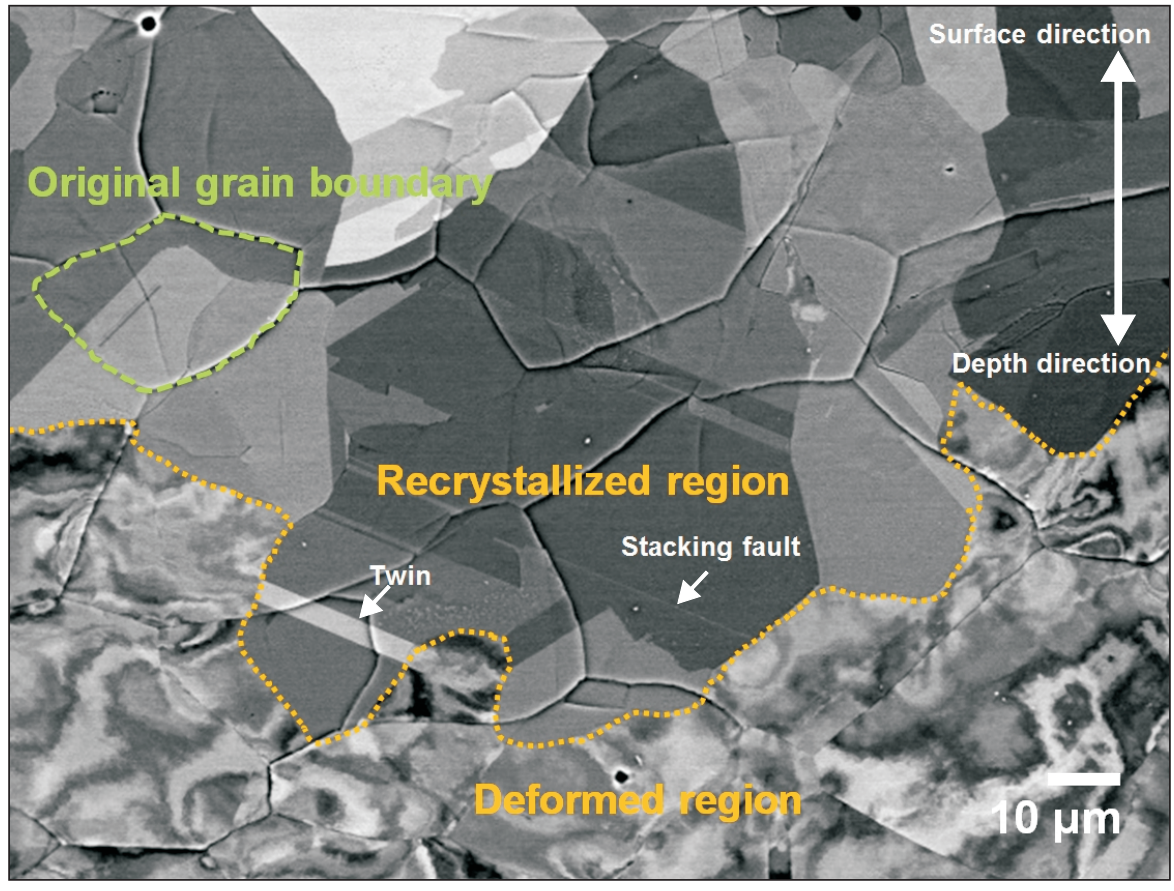

(a) This is an open-access article distributed under the terms of the Creative Commons Attribution Non-Commercial License (http://creativecommons.org/licenses/by-nc/4.0) which permits unrestricted noncommercial use, distribution, and reproduction in any medium, provided the original work is properly cited.

Copyrights @ 2018 by Korean Society of Microscopy 
Shot peening is a process that improves the mechanical properties of a metal or alloy target by plastic-deforming the target surface. Shot peening on the surface results in a linear decrease in the level of plastic deformation in the depth direction from the surface of the nickel. Accordingly, when the heat treatment is conducted, recrystallization process proceeds as a function of annealing time in the depth direction from the surface where the plastic deformation is severe. In this experiment, heat treatment was performed at $700^{\circ} \mathrm{C}$ for 5 minutes. The cross-sectional specimen was prepared by mechanical polishing before heat treatment and observed at the condition of the acceleration voltage of $30 \mathrm{kV}$, the probe current of $20 \mathrm{nA}$, and the working distance of $7.3 \mathrm{~mm}$ in COMPO mode using JEOL JSM-7000F field emission SEM. All areas at about $200 \mu \mathrm{m}$ deep from the surface were recrystallized. The above electron channeling contrast image was obtained at the border (orange dashed line) where the recrystallization ended in the 5 minute heat treatment. The electron channeling contrast imaging (ECCI) is a technique that shows the contrast induced by the difference in the backscattering yield of electrons according to crystal orientation (Lloyd, 1987). This technique is useful for observing the effect of plastic deformation because the yield of backscattering varies sensitively even with small angle difference. Therefore, recrystallized region and remaining area deformed by shot peening can be easily distinguished, as indicated by orange dashed line. The defects such as twins and stacking faults in the recrystallized grains were clearly observed as well. Furthermore, the original grain boundaries (green dashed line) of shot-peened nickel were thermally etched by the heat treatment for recrystallization (García de Andrés et al., 2002), standing out as a remarkable surface morphology. This morphology even remains after the recrystallization has occurred, so that the original grain boundaries can be easily observed as shown in the image. Consequently, the ECCI including original grain boundaries can clearly demonstrate the microstructural evolution during the recrystallization, and thus can provide better insight into the recrystallization behavior.

\section{CONFLICT OF INTEREST}

No potential conflict of interest relevant to this article was reported.

\section{ACKNOWLEDGMENTS}

This work was supported by the National Research Foundation of Korea(NRF) grant funded by the Korea government (MSIP) (2018R1A5A6075959).

\section{REFERENCES}

Lloyd G E (1987) Atomic number and crystallographic contrast images with the SEM: a review of backscattered electron techniques. Mineral. Mag. 51, 3-19.
García de Andrés C, Caballero F G, Capdevila C and San Martín D (2002) Revealing austenite grain boundaries by thermal etching: advantages and disadvantages. Mater. Charact. 49, 121-127. 